\title{
A Pneumatic Miniature Robotic Control System Based on Improved Single Neural Network PID Control
}

\section{Wen Li}

Hunan Institute of Science and Technology, Yueyang,

Hunan, 414006, China

E-mail: liwen07@sohu.com

\begin{abstract}
At present pneumatic miniature robotic control system usually adopts PID control strategy. The basic PID control strategy has its limitations. To cope with this problem, a lot of methods to optimize this strategy have been researched. In the paper an improved single neural network PID control strategy is put forward. The control strategy is a single neuron adaptive controller with adjusting weighting coefficient. Both simulation and experimental results indicate that this scheme is a feasible control method for the 3-dof pneumatic miniature robotic control system.
\end{abstract}

Keywords- Rubber actuator, Inchworm movement, single neural network, PID.

\section{INTRODUCTION}

Miniature robot used in minimally invasive treatment has become a hot spot of research on international [1-2]. In this medical application, the flexible peristalsis type robot based on pneumatic become preferred. It can enter the human body noninvasive or minimally. And, a 3-dof (Degree Of Freedom) miniature endoscopic inspection squirming robot system has been designed, which is driven by pneumatic rubber actuator. On this basis, the dynamic model of the robot has been built.

The researchers have found that research it is difficult to set its best parameters for the traditional PID control strategy when dealing with nonlinear controlled object by classical control theory. To cope with this problem, a lot of methods to optimize this strategy have been researched. Among them, single neural network PID control, with own learning and adaptive ability, not only has simple structure, and can adapt to the environment changes. It has strong robustness, and it is suitable for complicated modeling and control of nonlinear system. This study used an improved single neural network PID control strategy, and achieved good results.

\section{STRUCTURE OF THE PNEUMATIC AND FLEXIBLE MINIATURE ROBOT SYSTEM}

\section{A. Robot mobile mechanism}

Figure 1 shows the inchworm peristalsis principle.

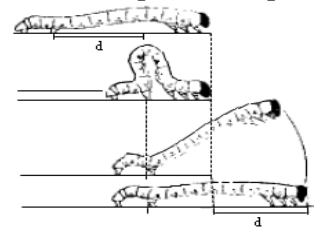

Figure 1. Earth-worm movement

\author{
Hong-Min Li
}

Hunan Institute of Science and Technology, Yueyang, Hunan, 414006, China E-mail: Lihmin171@sina.com

Miniature robot imitates this principle that has been verified in many literatures. It peristalsis and steering mobiles, through ultrasound positioning system in vitro and electricity - air system, which control a 3-dof gas chamber to make its inflatable or put gas [3-4], as figure 2 shows.
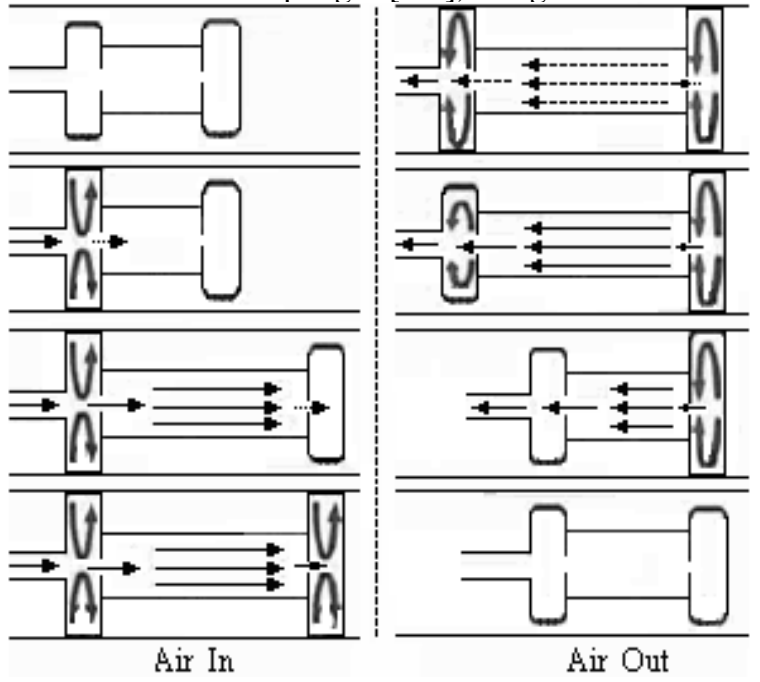

Figure2. Working Principle of the Proposed Moving Mechanism

From the figure 2, we can get the following information: in the initial state, each of the gas chamber is not inflatable state, and the drive is in free contracting. Then state cycles:

1. Holding unit after is clamping

Airbag after press the gas tube wall hung tightly after inflation. And this moment, the airbag before in holding unit before is in non-gas state.

2. Actuator is in elongation

Air drives three gas chambers. It makes actuator elongation. This result pushes holding unit before forward a step.

3. Holding unit before is clamping

Airbag before press the gas tube wall hung tightly after inflation.

4. Holding unit after loosens

The holding unit's gas valve is opened. Airbag put gas and it from gas tube wall.

5. Actuator shrinks

Actuator shrinks under the tension, which pushes holding unit after forward a step.

6. Restored to its original state

By completing this cycle, robot moved forward step. In this cycle, the sequence control for the gas chamber by air or put air shows as Figure 3. 


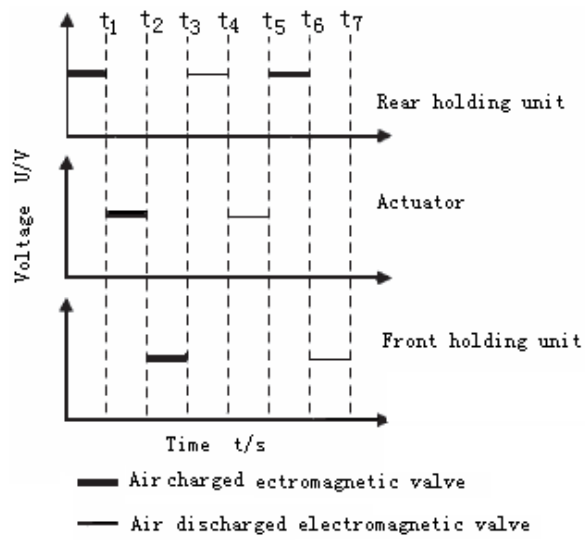

Figure.3. Time control orders for straight movement

Each valve that is connected with the gas chamber is controlled by voltage pulse signal.

The control method for linear movement is for: first, holding unit after is inflated, then, the computer in vitro controls holding unit and actuator to inflate or put gas according to the time sequence of $\mathrm{t} 1 \sim \mathrm{t} 7$.

\section{B. Structure of the Robot System}

The structure of the pneumatic miniature robotic system shows as figure 4 . Based on inchworm locomotion principle, the structure of robot is consist of holding unit before and after、actuator and sensors.

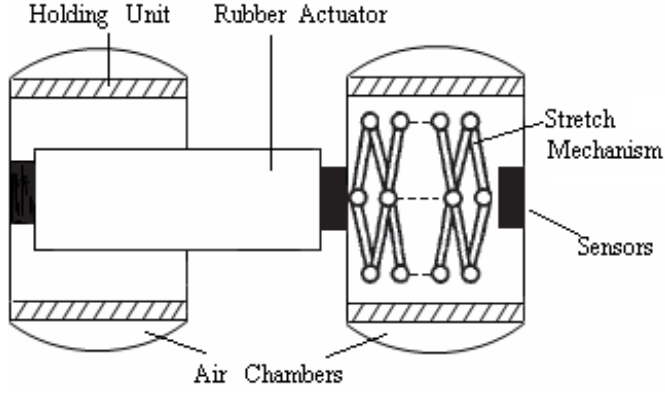

Figure 4. Structure of the robot

Holding unit before and after achieve clamping by means of pressing the airbag to the gas wall tightly.

Pneumatic rubber actuator is 3-dof elongation type. Its structure shows as figure 5.

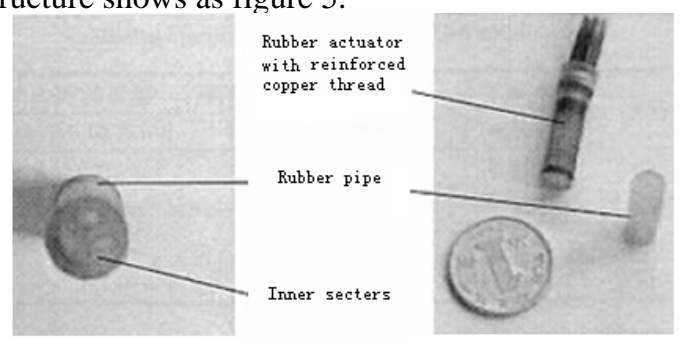

Figure 5. Rubber actuator

The rubber tube separates into three gas chambers that mutual into 120 degrees. Outer diameter of the actuator is $6 \mathrm{~mm}$ 、 diameter is $4 \mathrm{~mm}$ and long is $20 \mathrm{~mm}$. Thickness of fan-shaped rubber lumen is $1 \mathrm{~mm}$. Rubber layer in rubber tube accompanied by spiral fine fiber line. Fiber orientation and rubber presents certain angle, which can make the rubber actuator longitudinal elongation instead of horizontally increased. This actuator closes one side, and 3 air chambers of the other side connect tracheal respectively to control the press of each air chamber.

The actuator elongates along the axis when is added the same pressure to the air chamber. Instead, when just one chamber is added pressure or each of them is added different pressure, the actuator will bend and realize to steering finally. That is, through adjusting the pressure of the 3 air chambers, the robot realizes linear motion or steering movement.

Sensing institutions constituted by sensors and device component in general are for inspecting units, which are fixed in front scalable institutions. Through the retractable actuators, the sensors can extend holding unit before entering to orifice, to measure some parameters or diagnosis some disease [5].

\section{NONLINEAR MODELING OF THE ROBOT}

Analytical model shows as figure 6 [6].

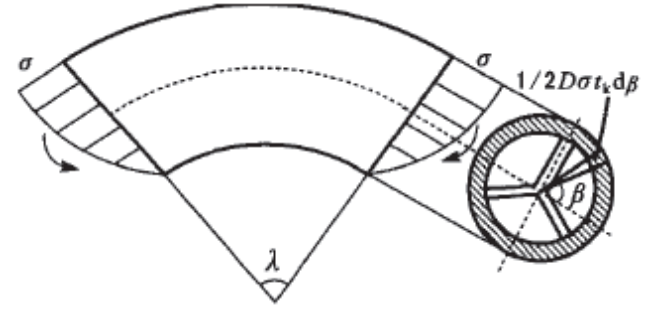

Figure 6. Analytic model of the actuator

Robots move straight when the three air chambers are added the same stress. Then, the stress of rubber wall can be expressed as $\sigma$ :

$$
\sigma=E \frac{\Delta L}{L+\Delta L}
$$

When linear deformation, elastic recovery force $(G)$ is defined as:

$$
G=E \frac{\Delta L}{L+\Delta L} \bullet 2 \pi \bullet \frac{D}{2} \bullet t_{k} \frac{L}{L+\Delta L}=\frac{\pi D t_{k} E L \Delta L}{(L+\Delta L)^{2}}
$$

The tension for actuator is expressed as:

$$
\begin{gathered}
F=P^{\prime} S_{0} \\
P^{\prime}=P_{1}-P_{0} \\
S_{0}=\frac{1}{4} \pi\left(D-2 t_{k}\right)^{2}-\frac{3}{2}\left(D-t_{k}\right) t_{f}
\end{gathered}
$$

By (5), volume of actuator is for:

$$
V=\left[\frac{1}{4} \pi\left(D-2 t_{k}\right)^{2}-\frac{3}{2}\left(D-t_{k}\right) t_{f}\right] L
$$
for:

System motion equation when robots move straight is

$$
F=G+f+v \frac{d \Delta L}{d t}+M \frac{d^{2} \Delta L}{d t^{2}}
$$

So dynamic characteristic equations when robots move straight by (3) and (7) can be represented as:

$$
\frac{\Delta L}{P^{\prime}}=\frac{1}{\pi D t_{k} E L} \cdot \frac{\omega_{n}^{2}}{s^{2}+2 \zeta \omega_{n} s+\omega_{n}^{2}}
$$


Where,

$$
\omega_{n}=\sqrt{\frac{\pi D t_{k} E L}{M}}, \zeta=\frac{v}{2 \sqrt{\pi D t_{k} E L M}}, E \text { is rubber }
$$

elastic modulus, $\Delta L$ is linear deformation, $L$ is original long of actuator, $\mathrm{D}$ is outside diameter of actuator, $t_{k}$ is thickness of shell, ${ }^{t}{ }_{f}$ is thickness of interior wall of air chamber, $f$ is friction force of air chamber interior, $\boldsymbol{V}$ is viscous damping coefficient,

$M$ is the mass of the micro-robot, $P^{\prime}$ is absolute pressure of air chamber, $P_{1}$ is the pressure of the num 1 air chamber, $P_{0}$ is atmospheric pressure, $S_{0}$ is the cross sectional area of actuator.

When just one chamber is added pressure or each of them is added different pressure, the actuator will bend and realizes to steering, and robots are bending movement. In this case, the system motion equation is for:

$$
N=I+N_{c}+v \frac{d \lambda}{d t}+J \frac{d^{2} \lambda}{d t^{2}}
$$

Where, $N$ is torsion moment produced by the force of gas expansion; $I$ is torsion moment produced by elastic recovery force of shell; $N_{c}$ is friction torque, $\lambda$ is angle of bend; $J$ is the rotary inertia of load.

In addition, the inherent frequency of systems based on cantilever beam model is for [7]:

$$
\omega=\sqrt{\frac{3 E I}{(N+0.236 M) L^{3}}}
$$

Finally, dynamic characteristic equations when robots move steering can be represented as:

$$
\frac{\lambda}{P^{\prime}}=\frac{S_{0} D L}{6 E L} \cdot \frac{\omega^{2}}{s^{2}+2 b \omega s+\omega^{2}}
$$

Where: $\quad b$ is the resistance coefficient of system.

\section{Single NEURAL NETWORK PID CONTROL SysteM}

\section{A. Miniature robotic control systems}

In general, the miniature robotic control system consists of pneumatic transmission system and computer control system.

Pneumatic transmission system consists of air compressor 、 air storage tank 、 flow control valves、 pressure regulator、3/2 and $21 / 2$ electromagnetic valve etc. There are 5 ventilation links connected airbag before 、 airbag aft er and 3 air chambers of actuator. Each link is connected by a 3/2 electromagnetic valve and a $2 / 2$ one. The $2 / 2$ electromagnetic valve connects air source which is in often broken state; the $3 / 2$ electromagnetic valve connects robot which is always to atmospheric. The relationship of the pneumatic pocket and magnetic valve are shown in table 1.

Table 1. The relationship of the pneumatic pocket and magnetic valve

\begin{tabular}{lccc}
\hline The preumatic pocket & pressure & maintain deflate \\
\hline $2 / 2$ magnetic valve & electrify outage & outage \\
$3 / 3$ magnetic valve & outage & outage electrify \\
\hline
\end{tabular}

Computer control system mainly consists of PC、A/D and D/A data collection equipment、Isolating amplifier drive circuit component. The computer adjusts the pressure of the air chamber to change the position of the mobile robot through judging duty ratio which is from constantly changing output PWM signal of feedback error signal. The figure 7 shows the Electro-pneumatic servo-control system [8].

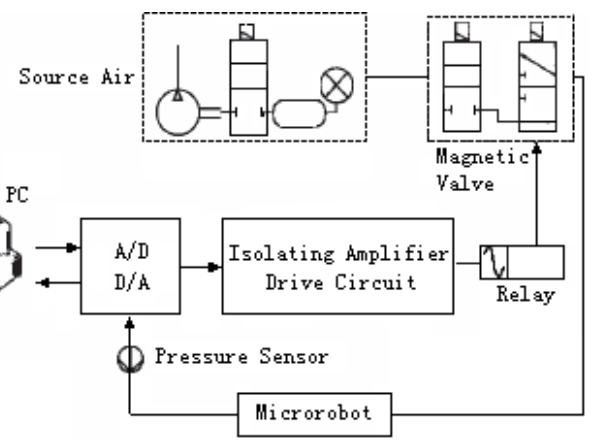

Figure 7. Electro-pneumatic servo-control system

B. The Improved Single Neural Network PID Control Method

Figure 8 shows the structure of the Single neural network adaptive PID control regulator [9].

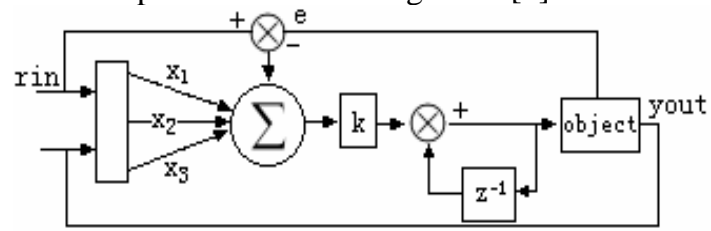

Figure 8. Structure of the Single neural network adaptive PID control regulator

Single neuron adaptive controller realizes its function by adjusting weighting coefficients. And the adjusting weighting coefficient is realized according to the Hebb learning rule with supervisory.

Control algorithm and learning algorithm is for:

$$
\begin{array}{r}
u(k)=u(k-1)+k \sum_{i=1}^{3} w_{i}(k) x_{i}(k) \\
w_{i}(k)=w_{i}(k) / \sum_{i=1}^{3}\left|w_{i}(k)\right| \\
w_{1}(k)=w_{1}(k-1)+\eta_{I} z(k) u(k) x_{1}(k) \\
w_{2}(k)=w_{2}(k-1)+\eta_{p} z(k) u(k) x_{2}(k) \\
w_{3}(k)=w_{3}(k-1)+\eta_{D} z(k) u(k) x_{3}(k)
\end{array}
$$

Where: $\quad x_{1}(k)=e(k), \quad x_{2}(k)=e(k)-e(k-1)$

$x_{3}(k)=\Delta^{2} e(k)=e(k)-2 e(k-1)+e(k-2)$

$z(k)=e(k), \eta_{D}, \eta_{P}, \eta_{I}$ are learning rates of differential, proportion and integral respectively, $\mathrm{K}$ is ratio of neural, $\mathrm{K}>0$.

Actual applications show that PID parameters of online learning and correction main are relate with $e(k)$ and $\Delta e(k)$.Therefore, we can modify the weighting coefficients: $\quad x_{i}(k)$ is modified to $e(k)+\Delta e(k)$, and $\Delta e(k)=e(k)+e(k-1)$. 
By using the modified above, weight coefficient online correction is not entirely based on neural network learning principle, but the actual experience of reference formulated.

\section{EXPERIMENTS AND SIMULATION}

Based on the nonlinear modeling of robot systems, we make reference signal: pulse signal; cycle is for $1 \mathrm{~s}$. Sampling time: $0.001 \mathrm{~s} . \eta_{\mathrm{D}}: 0.4 . \eta_{P}: 0.35$. $\eta_{I}: 0.4$. K: 0.12 . The simulation results of the relationship of input and output show as figure 9 .

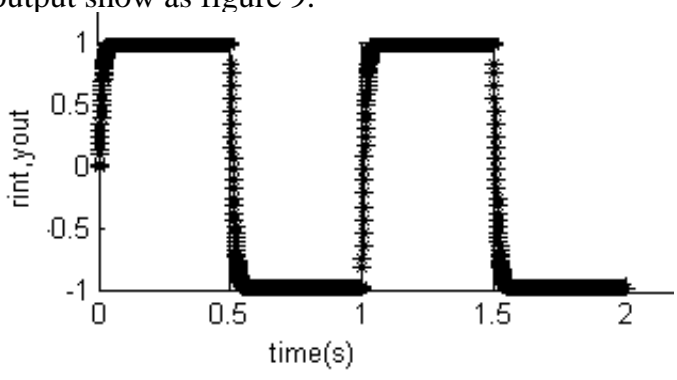

Figure 9. Simulation result of the position control system

When reference signals are laplace domain signal with different amplitude, the step-response curve of different sports mode of robot show as figure 10 and figure 11.

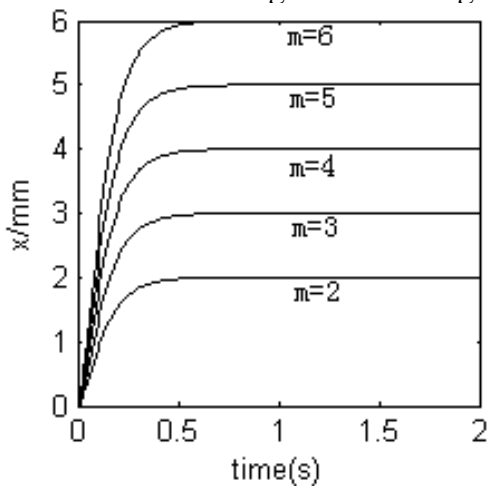

Figure 10. Experiment curves of the Linear movement system step-response

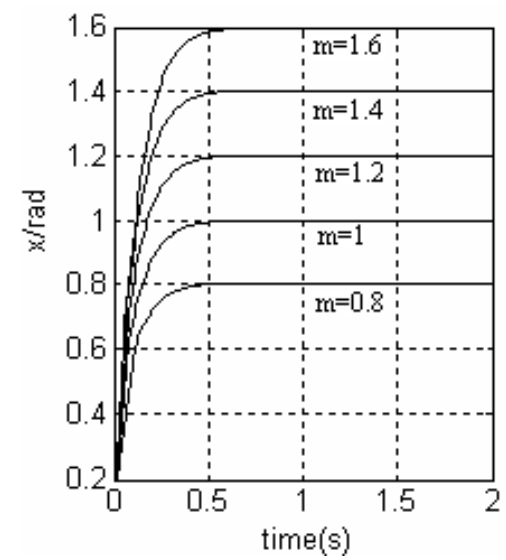

Figure 11. Experiment curves of the Steering mobile system step-response
From figure 10 and figure 11, we observe that this improved PID control method can track the target displacement fast and remain stable. The results of simulation mean that this method is feasible for the pneumatic miniature robotic control system.

\section{CONCLUSION}

This research uses a improved single neural network PID Control method in the 3-dof pneumatic miniature robotic control system. The results of simulation and experiment have shown good effects.

It proved that this method is feasible and it is expected that this control method has a good application prospect for pneumatic miniature robotic control system.

\section{ACKNOWLEDGMENT}

This research has been carried out with support of the Hunan Provincial Natural Science Foundation of China (10JJ2044).

\section{REFERENCES}

[1] Tondu B, Ippoloto S, Guiochet J. "A seven DOF robot-arm driven by pneumatic artificial muscles for humanoid robots," The International Journal of Robotics Research, Vol.24, 2005, pp. 257-274.

[2] Anthierens C, Libersa C, Touaibia M. "Micro robots dedicated to small diameter canalization exploration,” in Proc.IEEE International Conference on Intelligent Robots and Systems, Vol.1,2000,pp:480-485.

[3]Phee, L, Menciassi, A, Gorini, S, Wrnorio, G,Arena, A, Dario, P, “An Innovative Locomotion Principle for Minirobots Moving in the Gastrointestinal Tract”, Proc. of IEEE Int. Con\$ on Robotics \& Automation, pp.1125-1130

[4]Louis Phee, Dino Accoto, Arianna Menciassi,Cesare Stefanini, Maria Chiara Carrozza, Paolo Dario:“Analysis and Development of Locomotion Devices for the Gastrointestinal Tract”, IEEE Transactions on Biomedical Engineering, Vol: 49 Issue: 6 , Jun.2002, pp. 613-616.

[5] Suzumori K, Likura S, Tanaka H. "Applying a flexible microactuator to robotic mechanisms," IEEE International Conference on Robotics and Automation, Sacramento, CA.pp:21-27, April 1991.

[6] H.W. LIU. "Material Mechanics," Higher Education Publishing Company, Beijing, pp: 93-156,2004.

[7] J.L. WU. "Elastic Mechanics,” Tongji University Publishing Company, Shanghai, pp:140-167,2010.

[8] Jimwan Lim, Hyunjun Park, Sunmoo Moon. "Pneumatic Robot Based on Inchworm Motion for Small Diameter Pipe Inspection," in Proc.IEEE International Conference on Robotics and Biomimetics, pp:330-335, 2007. [9] J.K. LIU. "Advanced PID control And MATLAB simulation," Electronics Industry Publishing Company, Beijing, pp:153-159, 2004. 